\section{HEMOBILIA PÓS-TRAUMA}

\author{
HEMOBILIA POST TRAUMA
}

\section{Márcio Canavarros Serra, TCBC-MT ${ }^{1}$ \\ Janayna Cristhina Fortes ${ }^{2}$ \\ Roni Leonardo Teixeira ${ }^{2}$}

\section{INTRODUÇÃO}

O termo hemobilia, utilizado pela primeira vez em 1948 por Sanblom 1 , é causa rara de hemorragia digestiva alta, que ocorre pela formação de hematoma ou fístula arteriobiliar hepática. O trauma é responsável por $55 \%$ dos casos, e a manifestação clínica costuma ocorrer quatro a seis semanas depois. Caracteriza-se por hemorragia digestiva alta, dor abdominal e icterícia - síndrome de Quineke² ${ }^{2}$ Relatamos um caso de hemobilia pós-trauma em nosso serviço, e sua evolução satisfatória após a terapêutica adotada.

\section{RELATO DO CASO}

J.M.O., 58 anos, masculino, vítima de acidente por arma de fogo evoluiu com hemotórax à direita e lesão hepática tipo estrelar em lobo direito, suturada com categute cromado. Evidenciada também laceração do diafragma, suturada com náilon, não havendo outras lesões associadas. O hemotórax foi drenado e o paciente transferido do pronto-socorro, para nosso serviço no 7o pós-operatório, ainda com dreno de tórax. No 9o PO, evoluiu com hematêmese volumosa e melena, precedida de dor em flanco e fossa ilíaca direita, associado a icterícia, com colúria e acolia. A drenagem torácica apresentava-se com secreção serosanguinolenta de baixo débito. $\mathrm{O}$ paciente encontrava-se hemodinamicamente instável, com quedas constantes da hemoglobina e TAP baixo. A endoscopia evidenciou lesão bulbar em cicatrização, e a ultra-sonografia de abdome total evidenciou fístula arteriovenosa de alto débito em lobo hepático direito, confirmando o diagnóstico de hemobilia. Indicou-se a embolização da fístula por arteriografia hepática, a qual foi realizada com sucesso, confirmada por ultra-sonografia de controle três dias pós-procedimento. Quanto ao hemotórax, necessitou decorticação pulmonar à direita posteriormente, obtendo boa evolução pós-operatória.

\section{DISCUSSÃO}

Na propedêutica da hemobilia, o hemograma mostra queda do hematócrito e da hemoglobina, e há aumento de bilirrubinas, que pode ser flutuante (obstrução por coágulos). A endoscopia digestiva alta descarta outras causas de hemorragia do trato digestivo superior. A colangiografia endoscópica retrógrada com papilotomia drena coágulos e desobstrui as vias biliares, se necessário ${ }^{3}$.

Na ultra-sonografia abdominal com Doppler evidencia-se o local da fístula arteriobiliar (Figura 1), classificando-a quanto ao débito e orientando a arteriografia se-

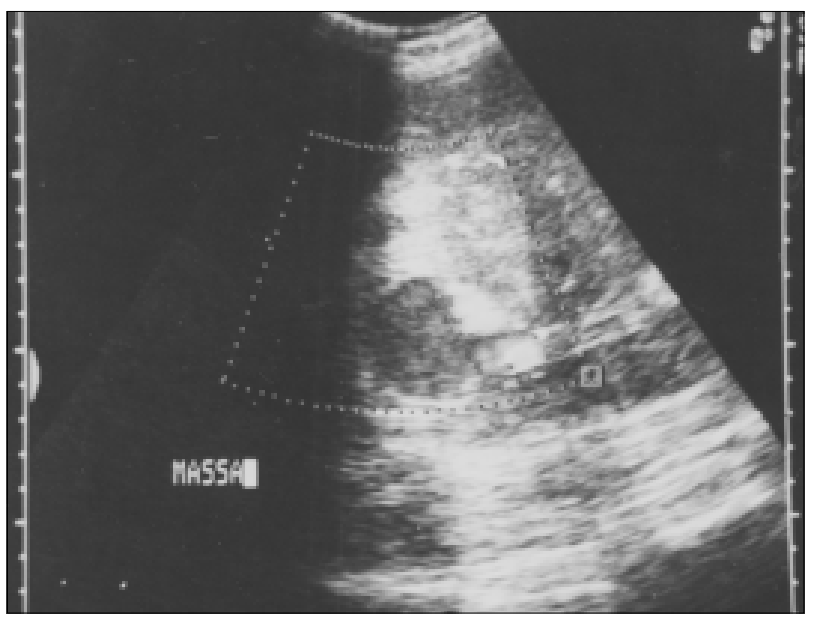

Figura 1 - Identificação por ultra-sonografia com Doppler de fistula em lobo direito de alto débito.

1. Professor Adjunto da Clínica Cirúrgica da Faculdade de Ciências Médicas do Hospital Universitário Júlio Muller da UFMT

2. Residente Cirurgia Geral do Hospital Universitário Júlio Muller da UFMT

Recebido em 03/12/2001

Aceito para publicação em 09/07/2002

Trabalho realizado no Departamento de Clínica Cirúrgica do Hospital Universitário Júlio Muller — Cuiabá-MT 
letiva da artéria hepática. Na suspeita de sangramento venoso deve-se realizar esplenoportografia ${ }^{3,4}$.

$\mathrm{O}$ tratamento conservador é feito com controle hidroeletrolítico e uso de antibióticos profiláticos. Arteriografias seriadas são realizadas quando ocorre perda sangüínea menor que $5 \%$ em 24 horas $^{3}$.

A ressecção hepática, é usada apenas nos casos de hemobilia traumática quando existe destruição tecidual importante, por tratar-se de cirurgia com alto índice de mortalidade (40\%). A arteriografia seletiva com embolização com Gel-Foam (Figura 2) ou álcool poly-vinyl após cateterização da artéria hepática soluciona de forma menos traumática o processo fistuloso, tem alta taxa de sucesso, diminuindo mortalidade para $20 \%$ 3,5.

$\mathrm{Na}$ falha da embolização, opta-se pela ligadura da artéria hepática, e este procedimento deve ser associado a drenagem das vias biliares, de hematomas existentes e do espaço subfrênico. Há relatos do uso de somatostatina para diminuir o fluxo sangüíneo visceral e facilitar a hemostasia local ${ }^{5}$.

Conclui-se que a arteriografia seletiva com embolização é o padrão-ouro, já que traz resolução com menor risco para a vida do paciente.

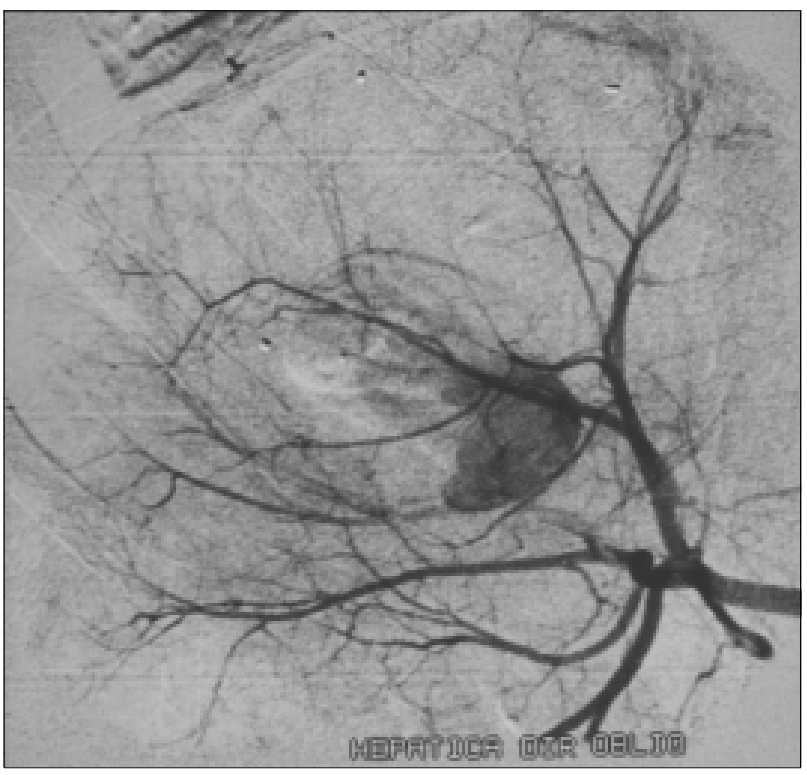

Figura 2 - Arteriografia da artéria hepática direita, com identificação da fístula e embolização com Gel-Foam.

\begin{abstract}
Our objective is to report a case of a patient, with a thoraco-abdominal gunshot wound with right hemothorax and liver lesion in the right lobe. The liver and the diafragm were sutured and the chest was drained. On the $9^{\text {th }}$ post operative day the patient had hematemesis, jaundice and pain in the right upper quadrant of the abdomen. The abdominal ultrasound image with Doppler, revealed arteriobiliary fistulae. The diagnosis of hemobilia was made and the patient underwent embolization of the fistulae by liver arteriography.
\end{abstract}

Key Words: Hemobilia post trauma; Arteriobiliary fistulae.

\title{
REFERÊNCIAS
}

1. Sanblom P. Haemobilia. In: Blumfart LH. Surgery of the liver and biliary tract, $1^{\mathrm{a}}$ ed. Churchill-Livingstone, Edinburg, 1988.

2. Adam R, Fabiani B, Bismuth H. Haemobilia. Surgery, 105:564, 1988.

3. Bober J, Vrzula A, Samet P et al. Post traumatic pseudoaneurysm of a anomalous right hepatic artery with arteriobiliary fistula and hemobilia. Rouzhl Chir, 78(9): 457-60, 1999.

4. Mizuni K, Itoh K, Monol T et al. Pancreaticobiliary arteriovenous malformation with common bile duct dilation in a patient with hemobilia. J. Clin Gastroenterol; 33 (1): 61-3, 2001.
5. Savassi-Rocha PR. Colecistoses, coleperitônio, peritonite biliar, hemobilia e parasitos. In: Dani R, Castro LP. Gastroenterologia Clínica, $3^{\mathrm{a}}$ ed. Guanabara-Koogan, Rio de Janeiro, 1988.

Endereço para correspondência:

Dr Márcio Canavarros Serra

Rua Sírio Libanesa 94 Apto 501 - Goiabeiras

78045-710 - Cuiabá-MT

E-mail:m.cserra@zaz.com.br 